\title{
A Double-Auction Mechanism for Distribution of Electrical Supply Capacities among Enterprises of an Industrial Park
}

\author{
Ariel M. Rocchi, Érica S. Fernandez and Jorge R. Vega, Member, IEEE
}

\begin{abstract}
Typically, an industry contracts a given electrical supply capacity to the energy distribution company for a relatively long time period. Such kind of contract is often expensive for the industry, because large supply capacities must be considered in order to avoid penalty fees due to an eventually high peak power demand that could occur even along a short time period. This paper proposes a dynamic strategy for simultaneously assigning the price and the fraction of power supply capacity to every industry located in an industrial park. The assignment strategy aims at reaching a joint benefit for all the involved enterprises (i.e., the industries and the energy distribution company). The proposed procedure involves 3 sequential algorithms. The strategy for supply capacity reassignment is based on a double-auction mechanism, which can be applied at time periods of arbitrary duration. The proposal is evaluated on the basis of two synthetic examples that involve different number of industries and electric power consumptions.
\end{abstract}

Index Terms - Double auction, Total capacity, Utilized capacity, Smart grids, Industrial park.

\section{INTRODUCCIÓN}

U n desafío importante para las redes eléctricas inteligentes es el proceso de toma de decisiones relacionadas con la comercialización de energía, el cual implica usualmente interacciones complejas entre los distintos actores y componentes de la red. En este contexto, conceptos de la Teoría de Juegos se utilizaron para determinar costos y beneficios de los agentes en función de sus estrategias. La Teoría de Juegos suele dividirse en juegos cooperativos y no cooperativos. Las aplicaciones principales se han centrado en los juegos cooperativos (o de coalición), habiéndose utilizado métodos de valor Shapley o valor Shapley bilateral -BVS-, Kernel y nucléolo. Los juegos no-cooperativos (o estratégicos) fueron menos utilizados y están asociados a una motivación individualista de los agentes, y no a las consecuencias de sus decisiones. La interacción entre los agentes participantes puede no ser de mutua colaboración, pero es posible inducirla mediante el diseño de mecanismos e incentivos [1].

Un enfoque basado en la Teoría de Juegos se adoptó para el control de fuentes/cargas individuales, lográndose mejorar la fiabilidad y la solidez de un sistema de potencia sin utilizar un control central [2]. Por otra parte, se propuso una nueva técnica basada en la Teoría de Juegos Cooperativa para gestionar la energía generada por turbinas eólicas y optimizar las ganancias [3]. También se estudiaron la compensación de potencia reactiva con el objetivo de optimizar la generación en parques eólicos [4], y el despacho económico de energía y reservas en mercados competitivos mediante algoritmos metaheurísticos $[5,6]$. Un modelo de juego estratégico se desarrolló para analizar un oligopolio dentro de un mercado de energía con varias restricciones a nivel de la red [7]. La planificación de la expansión de una red, tanto en transmisión como en generación, se estudió utilizando tres niveles de la Teoría de Juegos [8]. Un enfoque integral para la evaluación de los mercados de electricidad permitió investigar el impacto de varias restricciones sobre el equilibrio del mercado, utilizando para ello el sistema de prueba IEEE de 30 buses [9].

La comercialización de energía entre productores y consumidores se investigó recientemente en base a un modelo de subasta doble (SD) [10]. Para ello, se utilizó una estrategia de licitación que consideró las características y limitaciones técnicas de los diferentes dispositivos componentes de una micro-red eléctrica (por ejemplo, vehículos eléctricos, sistemas de micro-cogeneración, y bombas de calor). Los vehículos eléctricos híbridos conectables a la red (PHEVs) constituyen elementos clave en el emergente sistema de redes inteligentes. Por ejemplo, se estudiaron los complejos procesos de toma de decisiones de una serie de grupos de PHEV que buscan vender parte de su energía almacenada en un mercado energético [11], donde el precio de negociación que rige en el mercado de intercambio de energía entre los PHEVs y la red se determinó utilizando un mecanismo de SD. Para resolver el juego, se propuso un algoritmo basado en la dinámica de mejor respuesta, con el cual los grupos PHEVs pueden alcanzar un punto de equilibrio de Nash. Las SD han sido ampliamente utilizadas en los mercados de acciones, bonos y divisas [12].

El comercio entre micro-redes eléctricas interconectadas se investigó en base a un mecanismo de SD combinatoria, utilizándose algoritmos híbridos que combinan algoritmos genéticos con métodos de optimización por enjambre de partículas [13]. Otros autores propusieron una SD distribuida asistida por blockchain para facilitar el comercio de energía entre pares y garantizar la seguridad de los datos involucrados en la transacción. Este planteo apuntó a mitigar algunas desventajas de la gestión tradicional de transacciones basada en organizaciones centralizadas, como por ejemplo el alto costo de operación, la baja transparencia y el riesgo potencial de modificación de datos $[14,15]$. 
Las SD pueden ser de tiempo continuo (SDTC) o de tiempo discreto (SDTD). Una SDTC permite intercambios en cualquier momento durante un período de negociación, y las operaciones generales de dicha subasta están compuestas de múltiples transacciones bilaterales. Por otro lado, en una SDTD (también llamada clearing-house o call-market), todos los operadores se mueven en un solo paso desde la asignación inicial de transacciones hasta la asignación final [16]. En la literatura se han presentado varios modelos y diseños basados en SD para abordar diferentes situaciones en entornos de licitación dinámica de mercados de comercialización de energía en micro-redes [10, 17]. En algunos trabajos, se desarrollaron algoritmos de SD aplicables a micro-redes interconectadas, por ejemplo utilizando métodos de aprendizaje reforzado (reinforcement learning) [18].

En una red de distribución eléctrica, la Capacidad de Suministro (CS) se define como la potencia máxima (en kVA o kW), que una Empresa Distribuidora de Energía (EDE) pone a disposición de un cliente durante un período de tiempo establecido por contrato. En la bibliografía especializada, la CS también se suele reportar como utilized capacity o total capacity. Este trabajo focaliza el estudio en el contexto de un parque industrial donde residen varias empresas. En este caso, la CS de la EDE se la entiende como aquella potencia máxima puesta a disposición del conjunto de industrias del parque, en el punto de entrega identificado en el extremo del Alimentador Principal (AP). En esta situación, las industrias participantes serán interpretadas como agentes racionales que poseen incentivos o motivaciones disímiles; y la CS se la acepta lo suficientemente flexible como para poder ser negociada entre los agentes participantes del juego.

El presente trabajo utiliza varios conceptos del artículo de McAfee [19], quien desarrolló un protocolo de SD compatible con incentivos de estrategia dominante, el cual renuncia a la eficiencia de Pareto [16]. En comparación con otros trabajos relacionados a mercados en redes inteligentes $[3,9,20,21$, 22], y particularmente con respecto a [16, 19], en este artículo se pretende avanzar en los siguientes objetivos: 1) permitir a los participantes decidir estratégicamente el precio y las fracciones de CS que desean ofrecer o comprar; 2) optimizar la CS ociosa o inactiva de cada participante y la disponibilidad del AP; y 3) garantizar que se alcance un punto de equilibrio para juegos que muestran una discontinuidad en la función de utilidad, debido a la presencia de un modelo de subasta subyacente y a diferencia de los modelos clásicos que a menudo asumen utilidades continuas. En este contexto, se propone un marco estratégico que apunta a gestionar de manera eficiente las necesidades energéticas de un grupo de empresas radicadas en un parque industrial, donde la alimentación de energía eléctrica es provista por la EDE a través de un AP único. Se utiliza un juego no cooperativo para asignar precios y fracciones de CS, utilizando un mecanismo de SD donde cada participante elige estratégicamente la fracción de CS que desea vender o comprar. El incentivo para la EDE consistirá en maximizar sus beneficios, procurando optimizar la disponibilidad del AP. El incentivo para cada industria consistirá en minimizar el costo de la CS, buscando para ello reducir su CS ociosa. En tal sentido, el diseño y la implementación de un procedimiento iterativo procurarán atender a los incentivos mencionados y al mismo tiempo alcanzar convergencia y estabilidad de los resultados.

El resto del documento está organizado de la siguiente manera: la Sección II plantea el problema de distribución de la CS; la Sección III presenta el mecanismo de negociación propuesto para la asignación de precios y de fracciones de CS a los diferentes agentes participantes; en la Sección IV se desarrolla un caso de estudio simulado; y por último, las conclusiones del trabajo se detallan en la Sección V.

\section{Planteo del Problema}

Se asume la existencia de un parque industrial que alberga $N$ industrias con perfiles de consumo energético electrointensivos. En cada período de tiempo $(t)$, la industria $n(=$ $1, \ldots, N)$ tiene contratada una fracción de CS, CS $(n, t)$, de la capacidad de suministro de la EDE a través del AP, CS( $(t)$, de manera que:

$$
\operatorname{CS}(t)=\sum_{n=1}^{N} \operatorname{CS}(n, t)+\operatorname{CSR}(t)
$$

donde $\operatorname{CSR}(t)$ es la CS remanente en el período $t$ que dispone la EDE para una eventual asignación futura a otra industria o a un tercero externo al parque industrial. Es importante notar que deben cumplirse las siguientes restricciones:

$$
\begin{aligned}
\operatorname{CSR}(t) & \geq 0, \quad \forall t \\
\operatorname{CS}(n, t) & \geq \mathrm{P}(n, t), \quad \forall t, \forall n=1, \ldots, N
\end{aligned}
$$

donde $\mathrm{P}(n, t)$ representa la potencia eléctrica demandada por la industria $n$ en el período $t$. La violación de la restricción (2.a) implicaría una penalización a la EDE por su incapacidad para satisfacer la demanda; mientras que la violación de (2.b) originaría una penalización a la industria $n$. En contrapartida, cada industria preferirá contratar una $\operatorname{CS}(n, t)$ lo más cercana posible a $\mathrm{P}(n, t)$ para así reducir sus costos; mientras que la EDE pretenderá minimizar $\operatorname{CSR}(t)$ para maximizar sus ingresos por contratos.

Dado que toda industria puede tener un patrón de consumo variable en diferentes períodos de tiempo (por ej., semanal, mensual o estacional), es factible pensar que algunas industrias podrían preferir una estrategia dinámica para sus contratos de asignación de CS, implementados en períodos de tiempo más breves. De esta manera, cada industria procuraría regular en cada período de tiempo su $\operatorname{CS}(n, t)$ para que sea levemente superior a su $\mathrm{P}(n, t)$. A su vez, la EDE podría también estar interesada en esta estrategia de negocios, para poder fijar un precio por $\mathrm{kW}$ contratado más elevado cuando la CS contratada sea reducida, y adicionalmente obtener una mayor $\operatorname{CSR}(t)$ que le sirviera para continuar ofreciendo y vendiendo $\mathrm{CS}$ en vistas a maximizar sus ingresos.

Una posible solución al problema planteado puede abordarse con un enfoque de SD. En todo período de tiempo, cada industria conoce su situación energética particular; y en el siguiente período, elegirá estratégicamente la fracción de CS que estaría dispuesta a ofertar o a demandar, y los precios a los cuales pretenderá realizar sus transacciones. De igual manera, la EDE será otro agente participante de la SD, quien también evaluará y propondrá sus pretensiones de compraventa y precios de fracciones de CS. Teniendo en cuenta que 
tanto las industrias como la EDE estarán obligadas a participar en cada reapertura de la SD, entonces en el mecanismo de negociación que se presenta en la sección siguiente intervendrán $N+1$ agentes.

\section{MeCANiSmo de NegOciación}

La oferta y/o la demanda de la fracción de CS de potencia de un agente participante $j(=1, \ldots, J \leq N+1)$, así como los precios correspondientes, sólo pueden actualizarse al finalizar el período de tiempo actual $\left(t_{0}\right)$, y serán válidas para el período siguiente $(t)$. La duración de cada período la fija el ente regulador o subastador, quien supervisa, ejecuta y administra el procedimiento de negociación. Para poder definir la distribución de CS y precios entre los participantes, cada uno fija, antes del inicio del nuevo período $t$, una estrategia de modificación de su situación respecto al período actual $t_{0}$. Luego, se ejecuta un procedimiento que permite la asignación tanto de precios como de CS a los $N+1$ participantes, y este procedimiento se lo repite hasta alcanzar la solución que regirá para todo el nuevo período $t$.

La Fig. 1 presenta un esquema del procedimiento de asignación de precios y de fracción de CS (PAPYCS). Este procedimiento incluye tres algoritmos básicos de ejecución secuencial: 1) el algoritmo de SD (AlgSD), utilizado para determinar el precio al cual se realizarán las transacciones, 2) el algoritmo para asignar fracción de CS (AlgACS), el cual permite determinar, para cada pareja vendedor-comprador (seller-buyer) que calzó en la SD, la fracción de CS a la que posiblemente realizarán la transacción, y 3 ) el algoritmo para mejorar la eficiencia de las transacciones (AlgMET), el cual proveerá los valores finales de las fracciones de CS que mejor aprovechan las CS disponibles por los oferentes.

A continuación, se describe el PAPYCS. Cada ejecución del PAPYCS se identifica como un ciclo $c$, y previo a cada período $t$, se ejecutarán $\operatorname{los} C(t)$ ciclos necesarios para obtener un resultado. Al iniciarse cada nuevo período $t$, se actualizan los siguientes reportes: 1) la EDE publica sus Tablas de Oferta (TO) y Demanda (TD), y su CS actual, $\operatorname{CS}\left(j, t_{0}\right)$, e informa al subastador el precio de contratación correspondiente, $\left.\operatorname{Pr}\left(j, t_{0}\right) ; 2\right)$ cada industria informa al subastador y a la EDE su $\mathrm{P}(j, t)$ y su CS actual, $\operatorname{CS}\left(j, t_{0}\right)$, e informa al subastador el precio de contratación correspondiente, $\operatorname{Pr}\left(j, t_{0}\right)$, su TO $(j, t)$ y su $\operatorname{TD}(j, t) ;$ y 3$)$ al finalizar $\operatorname{los} C(t)$ ciclos, el subastador actualiza e informa la $\operatorname{CS}(j, t, C(t))$ y $\operatorname{Pr}(j, t, C(t))$ de cada participante $j$, y su precio, $\operatorname{Pr}(s b, t, C(t))$. Entonces, a través del PAPYCS, el subastador se ocupa de procesar los datos y de dar a conocer los resultados.

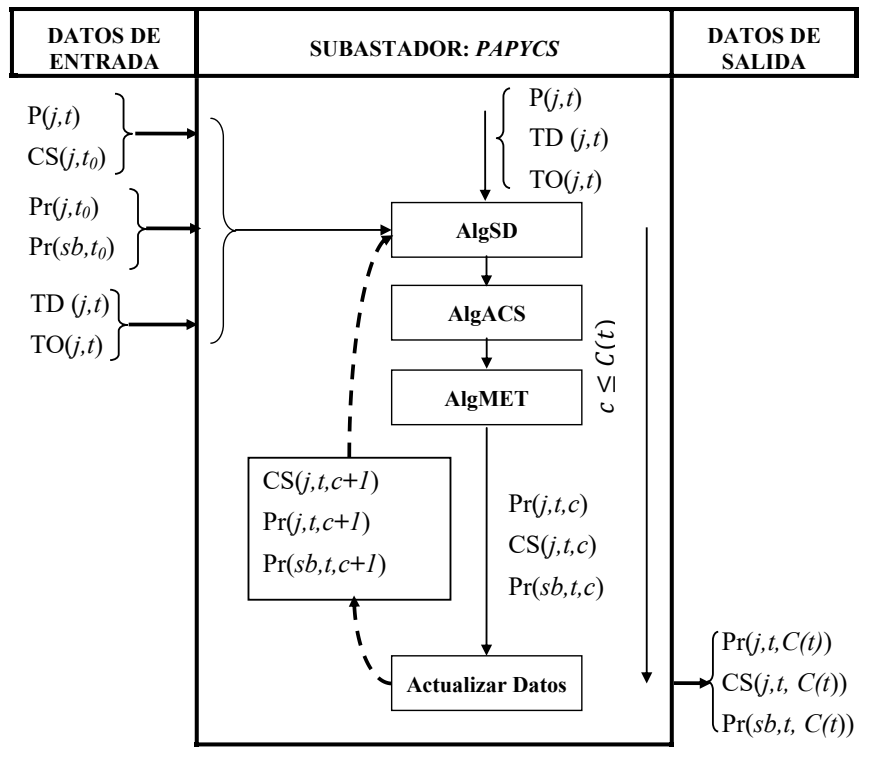

Fig. 1. PAPYCS: Procedimiento para asignar precios y fracción de CS al participante $j$, en el ciclo $c$ del período $t$.

Con el subastador conviven tres clases de datos: a) los que recibe al inicio de cada periodo $t$ (datos de entrada), b) los que debe informar una vez finalizada la SD, en el mismo periodo $t$ (datos de salida), y c) los que necesita PAPYCS para poder realimentarse y volver a ejecutarse: $\operatorname{CS}(j, t, c), \operatorname{Pr}(j, t, c)$, y $\operatorname{Pr}(s b, t, c)$. Además, en cada ciclo $c$, se asume que cada participante $j$ adopta una única posición (ya sea como vendedor o como comprador de fracción de CS). Cabe aclarar que, cada período $t$ esta signado por el valor de $\mathrm{P}(j, t)$.

\section{A. Datos de Entrada (Asociados al Periodo ' $t$ ')}

Cada participante $j$ dispone de la CS correspondiente al período $t_{0}$, y prevé requerir una potencia $\mathrm{P}(j, t)$ en el período $t$; y además, puede fijar una estrategia de oferta (o demanda) variada, explicitándola a través de sus TO y TD. Cada participante $j$ debe presentar las tablas actualizadas antes de que el subastador inicie PAPYCS. En efecto, al inicio de cada período $t$, cada participante informa al subastador: $\mathrm{P}(j, t)$; CS actual, $\operatorname{CS}\left(j, t_{0}\right)$; el precio de contratación, $\operatorname{Pr}\left(j, t_{0}\right)$; y las tablas: $\operatorname{TO}(j, t)$ y $\operatorname{TD}(j, t)$. En el PAPYCS, la variación de la CS de $j$, para un ciclo $c+1$, del período $t$, es:

$$
\Delta \operatorname{CS}(j, t, c+1)=\operatorname{CS}(j, t, c)-\mathrm{P}(j, t), \quad(j=1, \ldots, N+1)
$$

Entonces, el participante $j$ podrá ofrecer hasta un máximo de esa variación como $\mathrm{CS}$, si $\Delta \mathrm{CS}(j, t, c+1)>0$ (o bien, demandar como mínimo ese valor, si $\Delta \mathrm{CS}(j, t, c+1)<0)$. La expresión (3) insinúa también la magnitud de la CS ociosa o inactiva de cada participante $j$, en el ciclo $c$ del período $t$; mientras que el valor definitivo final quedará fijo al finalizar la ejecución del PAPYCS.

En la Tabla I, se presentan las TO y TD correspondientes al participante $j=1$, posicionado como vendedor, $\operatorname{TO}\left(j^{v}, t\right)$, o como comprador, $\operatorname{TD}\left(j^{c}, t\right)$, de fracciones de CS para el período $t$. Así, en la $\operatorname{TO}\left(1^{v}, t\right)$ hay $W$ ofertas postuladas por el participante $j=1$, quien ofrece vender $X s_{(w)}\left(1^{v}, t\right)$ fracciones 
de CS, a los precios $S_{(w)}\left(1^{v}, t\right)$, con $w=1, \ldots, W$; y en la $\operatorname{TD}\left(1^{c}, t\right)$ hay $Q$ ofertas postuladas por el mismo participante $j$ $=1$, quien ofrece comprar $X b_{(q)}\left(1^{c}, t\right)$ fracciones de CS, a los precios $b_{(q)}\left(1^{c}, t\right)$, con $q=1, \ldots, Q$. Nótese que las dimensiones $W$ y $Q$ pueden ser iguales o diferentes, y pueden además ser modificadas antes de que el subastador ejecute PAPYCS para obtener los valores correspondientes al nuevo período $t$.

TABLA I.

TO Y TD DEl PARTICIPANTE $j=1$ (PARA El PERÍOdo $t$ )

\begin{tabular}{cccc}
\hline \hline \multicolumn{2}{c}{$\mathrm{TO}\left(1^{v}, t\right)$} & \multicolumn{2}{c}{$\mathrm{TD}\left(1^{c}, t\right)$} \\
\hline$X s_{(1)}\left(1^{v}, t\right)$ & $s_{(1)}\left(1^{v}, t\right)$ & $b_{(1)}\left(1^{c}, t\right)$ & $X b_{(1)}\left(1^{c}, t\right)$ \\
$X s_{(2)}\left(1^{v}, t\right)$ & $s_{(2)}\left(1^{v}, t\right)$ & $b_{(2)}\left(1^{c}, t\right)$ & $X b_{(2)}\left(1^{c}, t\right)$ \\
$:$ & $:$ & $:$ & $:$ \\
$X s_{(W)}\left(1^{v}, t\right)$ & $s_{(W)}\left(1^{v}, t\right)$ & $b_{(Q)}\left(1^{c}, t\right)$ & $X b_{(Q)}\left(1^{c}, t\right)$ \\
\hline \hline
\end{tabular}

Además, el subastador ingresa al PAPYCS su $\operatorname{Pr}\left(s b, t_{0}\right)$.

\section{B. Datos de Entrada (Asociados al Ciclo 'c')}

El valor hallado en (3) es de gran importancia para el PAPYCS, ya que define el rol -como vendedor o compradordel participante $j$ en cada ciclo $c$ del período $t$. Para $c=1$ se cumple: $\quad \operatorname{CS}\left(j, t_{0}\right)=\operatorname{CS}(j, t, 1), \quad \operatorname{Pr}\left(j, t_{0}\right)=\operatorname{Pr}(j, t, 1), \quad \mathrm{y}$ $\operatorname{Pr}\left(s b, t_{0}\right)=\operatorname{Pr}(s b, t, 1)$.

\section{Algoritmo de Subasta Doble}

De acuerdo al protocolo PMD [16], cada participante declara sus precios como comprador $\left(b_{1}, \ldots, b_{M}\right)$ y como vendedor $\left(s_{1}, \ldots, s_{R}\right)$. Estos precios se arreglan en una matriz similar a la de la Tabla I, pero incluyendo sólo a aquellos valores que la Ec. (3) habilite como válidos para cada participante $j$, en el ciclo $c$ del período $t$. En consecuencia, se obtiene la matriz Ventas-Compras de la Tabla II. En este trabajo, el ordenamiento de los precios se elabora a partir de todos los precios declarados en la TO o la TD de la Tabla I. Los componentes de la Tabla II, deben cumplir:

$$
\begin{aligned}
& s_{(1)}\left(j^{v}, t, c\right)<s_{(2)}\left(j^{v}, t, c\right)<\ldots<s_{(R)}\left(j^{v}, t, c\right) \\
& b_{(1)}\left(j^{c}, t, c\right)>b_{(2)}\left(j^{c}, t, c\right)>\ldots>b_{(M)}\left(j^{c}, t, c\right)
\end{aligned}
$$

TABLA II.

MATRIZ DE VENTAS-COMPRAS PARA LOS PARTICIPANTES $j=1, \ldots, N+1$ (EN EL CICLO $c$ DEL PERÍODO $t$ )

\begin{tabular}{cccc}
\hline \hline \multicolumn{2}{c}{$\mathrm{TO}\left(j^{v}, \mathrm{t}, \mathrm{c}\right)$} & \multicolumn{2}{c}{$\mathrm{TD}\left(j^{c}, \mathrm{t}, \mathrm{c}\right)$} \\
\hline$X s_{(1)}\left(j^{v}, t, c\right)$ & $s_{(1)}\left(j^{v}, t, c\right)$ & $b_{(1)}\left(j^{c}, t, c\right)$ & $X b_{(1)}\left(j^{c}, t, c\right)$ \\
$X s_{(2)}\left(j^{v}, t, c\right)$ & $s_{(2)}\left(j^{v}, t, c\right)$ & $b_{(2)}\left(j^{c}, t, c\right)$ & $X b_{(2)}\left(j^{c}, t, c\right)$ \\
$\vdots$ & $\vdots$ & $\vdots$ & $:$ \\
$X s_{(\mathrm{R})}\left(j^{v}, t, c\right)$ & $s_{(\mathrm{R})}\left(j^{v}, t, c\right)$ & $b_{(\mathrm{M})}\left(j^{c}, t, c\right)$ & $X b_{(\mathrm{M})}\left(j^{c}, t, c\right)$ \\
\hline \hline
\end{tabular}

En (4) se observa el ordenamiento inverso de precios para compradores y vendedores. Para simplificar la descripción del protocolo, se define a $b_{(\mathrm{M}+1)}\left(j^{c}, t, c\right)$ como el precio más bajo posible de los compradores, y a $s_{(\mathrm{R}+1)}\left(j^{v}, t, c\right)$ como el precio más alto posible de los vendedores. Además, se verifica que:

$$
b_{(\mathrm{M}+1)}\left(j^{c}, t, c\right)<s_{(\mathrm{R}+1)}\left(j^{v}, t, c\right)
$$

Entonces se puede hallar un valor $\mathrm{k}$, tal que:

$$
\begin{gathered}
b_{(\mathrm{k})}\left(j^{c}, t, c\right) \geq s_{(\mathrm{k})}\left(j^{v}, t, c\right) \\
b_{(\mathrm{k}+1)}\left(j^{c}, t, c\right)<s_{(\mathrm{k}+1)}\left(j^{v}, t, c\right)
\end{gathered}
$$

Dado que las primeras $\mathrm{k}$ ofertas de los compradores superan a lo pretendido por los vendedores, entonces existen como máximo $\mathrm{k}$ transacciones posibles de compra-venta de CS. Para ellas, se define el siguiente precio de negociación:

$$
\operatorname{Pr}_{0}(t, c)=\frac{1}{2}\left\{b_{(\mathrm{k}+1)}\left(j^{c}, t, c\right)+s_{(\mathrm{k}+1)}\left(j^{v}, t, c\right)\right\}
$$

Se propone el siguiente protocolo de asignación de precios: 1- Si: $s_{(\mathrm{k})}\left(j^{v}, t, c\right) \leq P r_{0}(t, c) \leq b_{(\mathrm{k})}\left(j^{c}, t, c\right)$, entonces los primeros $\mathrm{k}$ compradores y vendedores negocian al precio $P r_{0}(t, c)$, descartando los precios que fijaron en sus respectivas ofertas.

2-Si: $\quad P r_{0}(t, c)>b_{(\mathrm{k})}\left(j^{c}, t, c\right) \quad$ o bien $\quad$ si: $\quad P r_{0}(t, c)<$ $s_{(\mathrm{k})}\left(j^{v}, t, c\right)$, entonces los primeros $\mathrm{k}-1$ compradores $\mathrm{y}$ vendedores negocian como sigue: los compradores al precio $b_{(\mathrm{k})}\left(j^{c}, t, c\right)$, y los vendedores al precio $s_{(\mathrm{k})}\left(j^{v}, t, c\right)$. En este caso, el siguiente monto es pagado por el comprador pero no es cobrado por el vendedor: $\operatorname{Pr}(s b, t, c)=(\mathrm{k}-1) \times$ $\left[b_{(\mathrm{k})}\left(j^{c}, t, c\right)-s_{(\mathrm{k})}\left(j^{v}, t, c\right)\right]$. Ese monto sobrante es asignado al subastador, el cual es un agente externo que no desea comprar ni vender.

Si la Ec. (3) señala que todos los participantes $j$ deben estar del lado de la oferta en la Tabla II, entonces la EDE pasa inmediatamente del lado de la demanda en la Tabla II. También, la EDE formará parte de la demanda de la Tabla II cuando no disponga de CS para vender.

\section{Algoritmo para Asignar la Capacidad de Suministro}

En cada renglón de la Tabla II, y hasta la posición donde se produce el calce del ordenamiento matricial ( $\mathrm{k}$ o k-1) determinado por AlgSD, se calcula el promedio de las fracciones de CS, según:

$$
\begin{array}{r}
X P_{(i)}(t, c)=\frac{1}{2}\left[X s_{(i)}\left(j^{v}, t, c\right)+X b_{(i)}\left(j^{c}, t, c\right)\right] \\
{[i=1, \ldots, \mathrm{k}(\mathrm{o}: \mathrm{k}-1)]}
\end{array}
$$

Esta fracción de CS promedio representa un posible acuerdo al cual podría realizarse la transacción entre cada pareja vendedor-comprador formada en la $\mathrm{SD}$, identificada en la posición $i$, correspondiente al ciclo $c$ del período $t$, hasta la posición de calce $\mathrm{k}$ o $\mathrm{k}-1$. Una forma de organizar la información obtenida con estos valores promedio es a través de una matriz. Como ejemplo, en la Tabla III se supone que hay 4 participantes, donde los participantes $j=1\left(1^{v}\right)$ y $j=4$ $\left(4^{v}\right)$ se postulan como vendedores, y los participantes $j=2$ $\left(2^{c}\right)$ y $j=3\left(3^{c}\right)$ se postulan como compradores.

En definitiva, el AlgACS permite establecer un criterio para asignar las fracciones de CS a comercializar entre los diferentes participantes de la $\mathrm{SD}$, en el ciclo $c$ del período $t$, 
sin que dichas fracciones necesariamente coincidan con lo pretendido originalmente por cada participante $j$.

TABLA III

FRACCIÓN PROMEDIO DE CS POR PAREJA VENDEDOR-COMPRADOR

\begin{tabular}{cccc}
\multicolumn{4}{c}{ (EN EL CICLO $c$ DEL PERIODO $t$ ) } \\
\hline \hline$i$ & Vendedor & $X P_{(i)}$ & Comprador \\
\hline$i=1$ & 1 & $X P_{(1)}(t, c)=0.75$ & 2 \\
$i=2$ & 1 & $X P_{(2)}(t, c)=1.50$ & 3 \\
$i=3$ & 4 & $X P_{(3)}(t, c)=3.50$ & 2 \\
$i=4$ & 1 & $X P_{(4)}(t, c)=3.50$ & 3 \\
$i=5$ & 1 & $X P_{(5)}(t, c)=5.50$ & 2 \\
$i=6$ & 4 & $X P_{(6)}(t, c)=6.00$ & 3 \\
$:$ & $:$ & $:$ & $:$ \\
$i=\mathrm{k}$ & $\ldots$ & $\ldots$ & $\cdots$ \\
$(\mathrm{o}: \mathrm{k}-1)$ & $\cdots$ & & \\
\hline \hline
\end{tabular}

\section{E. Algoritmo de Mejora de la Eficiencia de Transacciones}

Este algoritmo está diseñado para identificar y determinar los valores (o la combinación de valores) $X P_{(i)}(t, c)$ que mejor aprovechan las CS disponibles por los vendedores y que serán efectivamente asignadas a cada participante $j$ en cada ciclo $c$. Para ello, a partir de la Tabla III, se ejecutan los pasos que se detallan a continuación.

Paso 1. Con todas las combinaciones posibles de $X P_{(i)}(t, c)$ del vendedor $j^{v}$ de la Tabla III, se arma la matriz $\mathbf{M}_{j}\left(2^{v(j)} \times\right.$ $v(j)$ ), donde $v(j)$ es el número total de participaciones de $j^{v}$. Luego, se calculan los vectores de dimensiones $\left(2^{v(j)} \times 1\right)$ : a) $\mathbf{s}_{j}$, como la suma de las componentes de cada fila de la matriz $\mathbf{M}_{j}$; y b) $\mathbf{r}_{j}$, por diferencia entre el valor obtenido en la Ec. (3) por el participante $j^{v}$ y el valor de $\mathbf{s}_{j}$ correspondiente. Por ejemplo, en la Tabla III el vendedor $1^{v}$ participa 4 veces (filas $1,2,4,5)$, con los siguientes valores de $X P_{(i)}(t, c):\{0.75$, $1.50,3.50,5.50\}$; y se arma entonces la matriz $\mathbf{M}_{1}\left(2^{4} \times 4\right)$ y el vector $\mathbf{s}_{1}\left(2^{4} \times 1\right)$ [ver Tabla IV]. Como el resultado de la Ec. (3) para el vendedor $1^{v}$ es 2.57 , entonces se puede calcular $\mathbf{r}_{1}\left(2^{4} \times 1\right)$ [última columna de la Tabla IV].

Paso 2. Identificar los valores de $X P_{(i)}(t, c)$ de la fila de $\mathbf{M}_{j}$ correspondiente al menor valor positivo del vector $\mathbf{r}_{j}$. En el ejemplo, son los valores $\{1.50,0.75\}$ de la fila 4 , que sugieren identificar a las filas $i=1$ e $i=2$ de la Tabla III como habilitadas para la transacción comercial (tratos Ventas-Compras efectivos); mientras que no lo serían los casos de las filas $i=4 \mathrm{e}$ $i=5$. Si existiera un mínimo repetido del vector $\mathbf{r}_{j}$ (en 2 o más filas), entonces: i) para cada una de esas filas, en la Tabla III seleccionar aquella que tenga el valor $i$ más grande; y ii) de esos valores $i$ seleccionados, elegir el menor para identificar la transacción comercial finalmente habilitada.

Paso 3. Repetir los Pasos 1 y 2 para cada vendedor $j^{v}$.

Paso 4. Redefinir la Tabla III seleccionando en ella los tratos Ventas-Compras efectivos que ya fueron identificados en cada Paso 2 para cada vendedor $j^{v}$. Para el ejemplo, admitamos obtener la Tabla V, la cual contiene los valores que hacen eficientes las transacciones.

\section{F. Actualización de Valores}

A partir de la Tabla $\mathrm{V}$, se procede de la siguiente manera: a) Para cada vendedor $j^{v}$, calcular: i) $\Delta \operatorname{CS}^{v}(j, t, c)$, como la suma de los $X P_{(i)}(t, c)$; y ii) $u^{v}(j, t, c)$, como el número de veces que se repite ese vendedor. Para el ejemplo, se obtiene: i) para el participante $1^{v}: \Delta \operatorname{CS}^{v}(1, t, c)=2.25$ y $u^{v}(1, t, c)=$ 2 , y ii) para el participante $4^{v}: \Delta \operatorname{CS}^{v}(4, t, c)=9.50 \mathrm{y}$ $u^{v}(4, t, c)=2$.

b) Para cada comprador $j^{c}$, calcular: i) $\Delta \mathrm{CS}^{c}(j, t, c)$, como la suma de los $X P_{(i)}(t, c)$; y ii) $u^{c}(j, t, c)$, como el número de veces que se repite ese comprador. Para el ejemplo, se obtiene: i) para el participante $2^{c}: \Delta \operatorname{CS}^{c}(2, t, c)=4.25$ y $u^{c}(2, t, c)=$ 2 , y ii) para el participante $3^{c}: \Delta \operatorname{CS}^{c}(3, t, c)=7.50 \mathrm{y}$ $u^{c}(3, t, c)=2$.

Luego, para cada participante $j$, en el ciclo final $C(t)$, del período $t$, se calculan:

$$
\begin{aligned}
& \operatorname{CS}(j, t, C(t))=\operatorname{CS}\left(j, t_{0}\right)-\sum_{c=1}^{C(t)} \Delta \operatorname{CS}^{v}\left(j^{v}, t, c\right)+ \\
& \left.+\sum_{c=1}^{C(t)} \Delta \operatorname{CS}^{c}\left(j^{c}, t, c\right)\right) \\
& \operatorname{Pr}(j, t, C(t))=\operatorname{Pr}\left(j, t_{0}\right) \\
& +\sum_{c=1}^{C(t)} \operatorname{Pr}_{0}(t, c) \times u^{v}\left(j^{v}, t, c\right)- \\
& \text { - } \sum_{c=1}^{C(t)} P r_{0}(t, c) \times u^{c}\left(j^{c}, t, c\right)
\end{aligned}
$$

Y para el caso del Subastador:

$$
\operatorname{Pr}(s b, t, C(t))=\operatorname{Pr}\left(s b, t_{0}\right)-\sum_{c=1}^{C(t)} \sum_{1}^{j=N+1} \operatorname{Pr}(j, t, c)
$$

Cabe aclarar que para el vendedor $j^{v}$, si se cumple:

$$
\begin{array}{r}
0 \leq \Delta \operatorname{CS}^{v}\left(j^{v}, t, c+1\right)<\min X s_{(W)}\left(j^{v}, t, c\right): \\
/ X s_{(W)}\left(j^{v}, t, c\right) \in \operatorname{TO}(j, t)
\end{array}
$$

\begin{tabular}{|c|c|c|c|c|c|c|}
\hline \multicolumn{5}{|c|}{ Matriz $\mathbf{M}_{1}$} & \multirow[t]{2}{*}{$\mathbf{s}_{1}$} & \multirow[t]{2}{*}{$\mathbf{r}_{1}$} \\
\hline & 1 & 2 & 3 & 4 & & \\
\hline 1 & 0 & 0 & 0 & 0 & 0.00 & 2.57 \\
\hline 2 & 0 & 0 & 0 & 0.75 & 0.75 & 1.82 \\
\hline 3 & 0 & 0 & 1.50 & 0 & 1.50 & 1.07 \\
\hline 4 & 0 & 0 & 1.50 & 0.75 & 2.25 & 0.32 \\
\hline 5 & 0 & 3.50 & 0 & 0 & 3.50 & -0.93 \\
\hline 6 & 0 & 3.50 & 0 & 0.75 & 4.25 & -1.68 \\
\hline 7 & 0 & 3.50 & 1.50 & 0 & 5.00 & -2.43 \\
\hline 8 & 0 & 3.50 & 1.50 & 0.75 & 5.75 & -3.18 \\
\hline 9 & 5.50 & 0 & 0 & 0 & 5.50 & -2.93 \\
\hline 10 & 5.50 & 0 & 0 & 0.75 & 6.25 & -3.68 \\
\hline 11 & 5.50 & 0 & 1.50 & 0 & 7.00 & -4.43 \\
\hline 12 & 5.50 & 0 & 1.50 & 0.75 & 7.75 & -5.18 \\
\hline 13 & 5.50 & 3.50 & 0 & 0 & 9.00 & -6.43 \\
\hline 14 & 5.50 & 3.50 & 0 & 0.75 & 9.75 & -7.18 \\
\hline 15 & 5.50 & 3.50 & 1.50 & 0 & 10.50 & -7.93 \\
\hline 16 & 5.50 & 3.50 & 1.50 & 0.75 & 11.25 & -8.68 \\
\hline
\end{tabular}

entonces $j$ ya no participará en la siguiente ejecución del PAPYCS, en el próximo ciclo $c$ del período $t$.

TABLA IV

MATRIZ DEL VENDEDOR $1^{v}, \mathrm{M}_{1}$ Y VECTORES $\mathbf{s}_{1}$ Y $\mathbf{r}_{1}$ ASOCIADOS (EN EL CICLO $c$ DEL PERÍODO $t$ ) 
TABLA V

VENTAS-COMPRAS: TRATOS EFECTIVOS (EN EL CICLO $c$ DEL PERÍODO $t$ )

\begin{tabular}{cccc}
\hline \hline $\mathrm{i}$ & Vendedor & $\mathrm{XP}_{(\mathrm{i})}$ & Comprador \\
\hline$i=1$ & 1 & $X P_{(1)}(t, c)=0.75$ & 2 \\
$i=2$ & 1 & $X P_{(2)}(t, c)=1.50$ & 3 \\
$i=3$ & 4 & $X P_{(3)}(t, c)=3.50$ & 2 \\
$i=6$ & 4 & $X P_{(6)}(t, c)=6.00$ & 3 \\
$:$ & $:$ & $:$ & $:$ \\
$i=\mathrm{k}$ & $\ldots$ & $\ldots$ & $\cdots$ \\
$(\mathrm{o}: \mathrm{k}-1)$ & $\cdots$ & & $\cdots$ \\
\hline \hline
\end{tabular}

\section{G. Datos de Salida (Asociados al Ciclo 'c')}

Finalmente, para el período $t$ y ciclo $c$, el procedimiento propuesto realimenta la información para una nueva ejecución del PAPYCS, aumentando en uno el número de ciclos. El PAPYCS deja de ejecutarse cuando en la Tabla II ya no existen participantes vendedores.

\section{H. Datos de Salida (Asociados al Periodo 't')}

Los resultados obtenidos del PAPYCS son recogidos y analizados por el subastador. Si los resultados del ciclo $c+1$ son idénticos a los obtenidos en el ciclo $c$, el subastador detiene al PAPYCS e informa a cada participante $j$ en el período $t$ los valores: $\operatorname{Pr}(j, t, C(t)), \operatorname{Pr}(s b, t, C(t)), \quad \mathrm{y}$ CS $(j, t, C(t))$, identificando además el valor de $c=C(t)$.

A continuación, se desarrolla un seudo-código descriptivo de los algoritmos detallados anteriormente. Estos seudocódigos describen la ejecución de un ciclo del PAPYCS, proveyendo una descripción genérica de los bloques representados en la Fig. 1.

\section{Seudo-Código de un Ciclo de PAPYCS}

Datos de entrada: $\mathrm{P}(j, t), \operatorname{TD}(j, t), \mathrm{TO}(j, t) . \mathrm{Y}$ además, para $c=1$ : $\mathrm{CS}\left(j, t_{0}\right), \operatorname{Pr}\left(j, t_{0}\right), \operatorname{Pr}\left(s b, t_{0}\right)$.

\section{Inicio de AlgSD}

1. Declarar los precios de cada $j$ como $j^{c}$ y como $j^{v}: s_{(r)}$ y $b_{(m)}$.

2. Armar las TO y TD, respetando la Ec. (3), y ordenando según las Ecs. (4.a) y (4.b). Obtener la Tabla II.

3. Comparar las columnas de $s_{(r)}$ y $b_{(m)}$ hasta hallar la posición de calce, $\mathrm{k}$, que verifique las Ecs. (6).

4. Calcular el precio de negociación $P r_{0}$ con la Ec. (7).

5. SI: $s_{(\mathrm{k})}\left(j^{v}\right) \leq P r_{0} \leq b_{(\mathrm{k})}\left(j^{c}\right)$, ENTONCES:

Los k primeros $j^{c}$ y $j^{v}$ negocian a $P r_{0}$.

SI: $\operatorname{Pr}_{0}>b_{(\mathrm{k})}\left(j^{c}\right)$ o $P r_{0}<s_{(\mathrm{k})}\left(j^{v}\right)$, ENTONCES:

Los (k-1) primeros $j^{c}$ negocian a $b_{(\mathrm{k})}\left(j^{c}\right)$ y los $(\mathrm{k}-1)$ primeros $j^{v}$ negocian a $s_{(\mathrm{k})}\left(j^{v}\right)$.

Asignar el monto sobrante al subastador: $\operatorname{Pr}(s b)$.

\section{Fin de AlgSD}

\section{Resultados de AlgSD transferidos a AlgACS \\ Tabla II.}

\section{Inicio de AlgACS}

1. Para cada posición $i$ de las TO y TD (Tabla II), calcular $X P_{(i)}$ con la Ec. (8).

2. Construir la Tabla III, incorporando $X P_{(i)}$ a la Tabla II.

Fin de AlgACS

\section{Resultados de AlgACS transferidos a AlgMET}

Tabla III

\section{Inicio de AlgMET}

1. Para cada vendedor $j^{v}(=1,2, \ldots)$ :

(i) Calcular las participaciones $j^{v}, v(j)$, a partir de la Tabla III.

(ii) Armar la matriz $\mathbf{M}_{j}$, del vendedor $j^{v}$ (Tabla IV).

(iii) Calcular los vectores $\mathbf{s}_{j}$ y $\mathbf{r}_{j}$ (Tabla IV).

(iv) Con $\operatorname{los} X P_{(i)}$ de la fila de $\mathbf{M}_{j}$ con menor valor positivo de $\mathbf{r}_{j}$, identificar las correspondientes filas $i$ en la Tabla III.

2. Armar la Tabla V a partir de los tratos ventas-compras efectivos determinados en el paso anterior.

Fin de AlgMET

\section{CAso de Estudio}

La implementación computacional de los algoritmos descriptos es compleja debido a que, en cada ciclo $c$, cada participante $j$ puede quedar (o no) involucrado en el PAPYCS. Además, en el caso de quedar involucrado, puede alternar (o no) su posicionamiento como vendedor o como comprador.

A continuación se presentan dos ejemplos sintéticos de dimensión reducida. El símbolo \$a representa a una unidad de valor arbitraria.

\section{A. Ejemplo 1}

La Fig. 2 muestra un esquema de las $N=3$ industrias participantes y el AP de la EDE $(j=4)$. Se asume que la EDE tiene una $\mathrm{CS}$ de $30[\mathrm{~kW}]$.

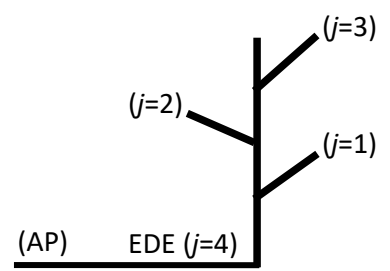

Fig. 2. Ej. 1. Esquema conceptual de conexiones de 4 participantes al $\mathrm{AP}(j=$ $1,2,3$ : industrias; $j=4$ : EDE).

Analicemos inicialmente el caso estándar, cuando no se dispone de ningún criterio de regulación o reasignación de fracciones de CS. La Fig. 3 indica el comportamientorequerimiento $\mathrm{P}(j, t)$ de cada participante $j$ en un período $t$. La potencia total, $\operatorname{PT}(t)$, la CS total provista por la $\mathrm{EDE}, \operatorname{CST}(t)$, y la CS inactiva del AP, $\operatorname{CSI}(t)$, se calculan según (ver Fig. 3):

$$
\begin{aligned}
& \operatorname{PT}(t)=\sum_{\mathrm{j}=1}^{\mathrm{N}} \mathrm{P}(j, t) \\
& \operatorname{CST}(t)=\sum_{j=1}^{\mathrm{N}} \operatorname{cs}(j, t, C(t)) \\
& \operatorname{cSI}(t)=\operatorname{CST}(t)-\operatorname{PT}(t)
\end{aligned}
$$

La Tabla VI detalla los valores de la Fig. 3. Las CS de las 3 industrias se mantienen constantes para los diferentes valores de $t$, porque usualmente cada industria contrata a la EDE una única CS anual (cota máxima de potencia que no debe ser superada para evitar penalizaciones). La EDE $(j=4)$ no interviene en la Tabla VI porque su potencia transaccional es nula en todos los intervalos de tiempo, es decir $\mathrm{P}(4, t)=0$. 


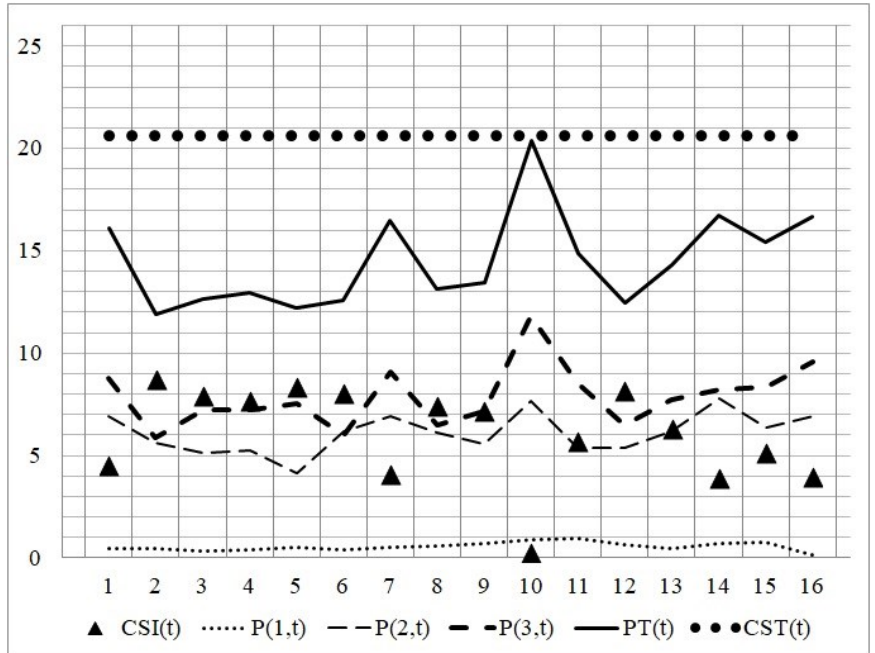

Fig. 3 . Ej. 1. Evoluciones de CSs y potencias a lo largo de 16 períodos. Caso de la CS contratada constante.

TABLA VI

EJ. 1. P $(j, t)$ Y CS $(j, t, C(t))$ EN [kW], POR PARTICIPANTE $j$ EN EL PERÍODO $t$

\begin{tabular}{ccccccccc}
\hline \hline$t$ & $\mathrm{P}(1, t)$ & $\mathrm{CS}(1, t, 1)$ & $\mathrm{P}(2, t)$ & $\mathrm{CS}(2, t, 1)$ & $\mathrm{P}(3, t)$ & $\mathrm{CS}(3, t, 1)$ & $\mathrm{PT}(t)$ & $\mathrm{CST}(t)$ \\
\hline 1 & 0.43 & 1.00 & 6.91 & 7.80 & 8.75 & 11.80 & 16.08 & 20.60 \\
2 & 0.46 & 1.00 & 5.60 & 7.80 & 5.85 & 11.80 & 11.90 & 20.60 \\
3 & 0.31 & 1.00 & 5.13 & 7.80 & 7.22 & 11.80 & 12.65 & 20.60 \\
4 & 0.40 & 1.00 & 5.27 & 7.80 & 7.26 & 11.80 & 12.93 & 20.60 \\
5 & 0.53 & 1.00 & 4.15 & 7.80 & 7.54 & 11.80 & 12.22 & 20.60 \\
6 & 0.39 & 1.00 & 6.17 & 7.80 & 6.01 & 11.80 & 12.57 & 20.60 \\
7 & 0.52 & 1.00 & 6.90 & 7.80 & 9.07 & 11.80 & 16.49 & 20.60 \\
8 & 0.58 & 1.00 & 6.10 & 7.80 & 6.48 & 11.80 & 13.16 & 20.60 \\
9 & 0.70 & 1.00 & 5.57 & 7.80 & 7.18 & 11.80 & 13.45 & 20.60 \\
10 & 0.90 & 1.00 & 7.65 & 7.80 & 11.79 & 11.80 & 20.35 & 20.60 \\
11 & 0.95 & 1.00 & 5.39 & 7.80 & 8.54 & 11.80 & 14.89 & 20.60 \\
12 & 0.64 & 1.00 & 5.35 & 7.80 & 6.46 & 11.80 & 12.45 & 20.60 \\
13 & 0.44 & 1.00 & 6.16 & 7.80 & 7.70 & 11.80 & 14.31 & 20.60 \\
14 & 0.72 & 1.00 & 7.77 & 7.80 & 8.22 & 11.80 & 16.71 & 20.60 \\
15 & 0.73 & 1.00 & 6.38 & 7.80 & 8.33 & 11.80 & 15.44 & 20.60 \\
16 & 0.15 & 1.00 & 6.94 & 7.80 & 9.56 & 11.80 & 16.66 & 20.60 \\
\hline \hline
\end{tabular}

A continuación, se analiza la aplicación del método propuesto, para el caso en que se permite una reasignación de fracciones de CS. Las Tablas VII y VIII muestran, respectivamente, las matrices de demandas, $\operatorname{TD}\left(j^{c}, t\right)$, y de ofertas, $\operatorname{TO}\left(j^{v}, t\right)$, de acuerdo a los criterios elegidos por cada participante $j$ y por la EDE.

TABLA VII

EJ. $1 . \operatorname{TD}\left(j^{c}, t\right)$ Y TO $\left(j^{v}, t\right)$, POR PARTICIPANTE $j$ EN EL PERÍODO $t$ $X b(\mathrm{~kW})-X s(\mathrm{~kW})-b(\$ \mathrm{a})-s(\$ \mathrm{a})$

\begin{tabular}{ccccccccccccc}
\hline \hline \multicolumn{2}{c}{ TD } & \multicolumn{2}{c}{ TO } & \multicolumn{2}{c}{ TD } & \multicolumn{2}{c}{ TO } & \multicolumn{2}{c}{ TD } & \multicolumn{2}{c}{ TO } \\
\multicolumn{2}{c}{$\left(1^{c}, t\right)$} & \multicolumn{2}{c}{$\left(1^{v}, t\right)$} & \multicolumn{2}{c}{$\left(2^{c}, t\right)$} & \multicolumn{2}{c}{$\left(2^{v}, t\right)$} & \multicolumn{2}{c}{$\left(3^{c}, t\right)$} & \multicolumn{2}{c}{$\left(3^{v}, t\right)$} \\
$b$ & $X b$ & $X s$ & $s$ & $b$ & $X b$ & $X s$ & $s$ & $b$ & $X b$ & $X s$ & $s$ \\
\hline 8.0 & 1.0 & 0.5 & 1.8 & 8.5 & 1.0 & 1.0 & 2.5 & 7.7 & 1.0 & 1.0 & 3.1 \\
6.6 & 4.0 & 2.0 & 2.4 & 7.0 & 4.0 & 2.0 & 3.0 & 6.3 & 4.0 & 2.0 & 3.7 \\
5.6 & 7.0 & 3.0 & 2.9 & 6.0 & 7.0 & 3.0 & 3.6 & 5.3 & 7.0 & 3.0 & 4.0 \\
4.6 & 10 & 4.0 & 3.4 & 5.0 & 10 & 4.0 & 4.1 & 4.3 & 10 & 4.0 & 4.5 \\
\hline \hline
\end{tabular}

TABLA VIII

EJ. $1 . \operatorname{TD}\left(4^{c}, t\right)$ Y TO $\left(4^{v}, t\right)$, PARA LA EDE EN EL PERÍODO $t$. $X b(\mathrm{~kW})-X s(\mathrm{~kW})-b(\$ \mathrm{a})-s(\$ \mathrm{a})$

\begin{tabular}{ccc}
\hline \hline$X s(\mathrm{o} X b)$ & $s$ & $b$ \\
\hline 3.00 & 2.60 & 6.50 \\
5.00 & 3.50 & 5.50 \\
7.00 & 5.00 & 5.00 \\
9.00 & 7.00 & 4.50 \\
11.00 & 7.70 & 4.00 \\
13.00 & 8.45 & 3.50 \\
15.00 & 9.00 & 3.00 \\
17.00 & 9.35 & 2.50 \\
19.00 & 9.50 & 2.00 \\
\hline \hline
\end{tabular}

En la Fig. 4 se observa el comportamiento de la $\operatorname{CST}(t)$ y la $\operatorname{CSI}(t)$, resultante de aplicar el PAPYCS, partiendo de $\operatorname{CS}\left(j, t_{0}\right)=0, y \operatorname{Pr}\left(j, t_{0}\right)=0$ para todo participante $j(=$ $1,2,3)$, y $\operatorname{Pr}\left(s b, t_{0}\right)=0$. Las Tablas IX y X detallan los valores de $\operatorname{CS}(j, t, C(t))$ y $\operatorname{Pr}(j, t, C(t))$ obtenidos con el PAPYCS, en cada período $t$. Un valor negativo indica una entrega de CS (o un pago monetario), y un valor positivo indica una adquisición de CS (o un cobro monetario). En ambas tablas, ya sea para $\operatorname{CS}(j, t, C(t))$ o $\operatorname{Pr}(j, t, C(t))$, la suma de sus valores por período $t$ debe ser cero. La Tabla X resume los valores de $\operatorname{Pr}(s b, t, C(t))$ que adquiere el subastador. En este trabajo se asume que el subastador no es un agente comercial; aun así, recibe el pago en cada período $t$, de acuerdo con [16].

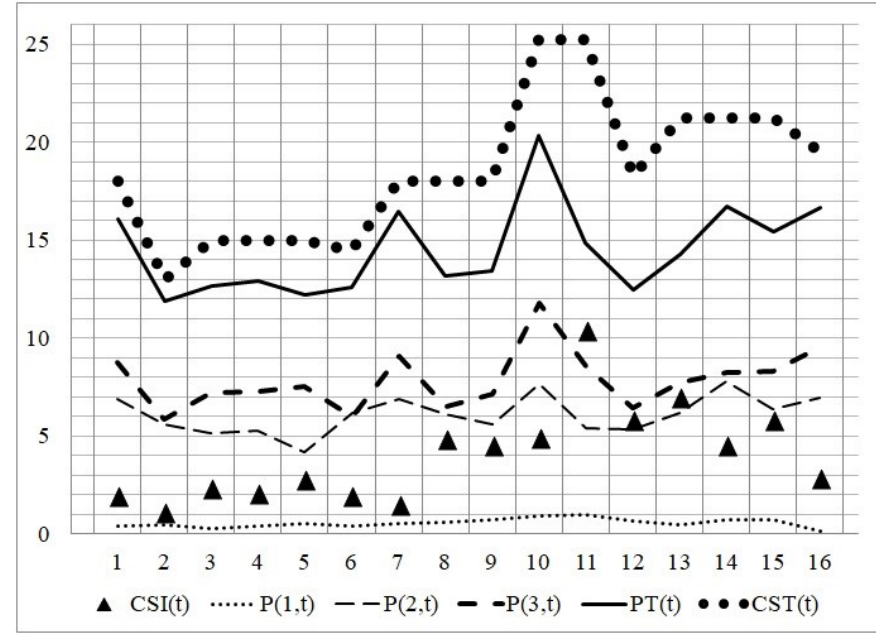

Fig. 4. Ej. 1. Evoluciones de CSs y potencias a lo largo de 16 períodos. Caso de fracciones de CS contratadas variables entre períodos $t$.

El valor de $\operatorname{CSR}(t)$ se calcula según (ver Ecs. (1) y (14)):

$$
\operatorname{CSR}(t)=\operatorname{CS}(t)-\operatorname{CST}(t)
$$

Los valores acumulados $(a c)$ de la $\operatorname{CSI}(t)$ y de la $\operatorname{CSR}(t)$, para todos los períodos $t$ analizados, se calculan a través de las siguientes expresiones (en $[\mathrm{kW}])$ :

$$
\begin{aligned}
& \operatorname{CSI}_{(a c)}=\sum_{1}^{t} \operatorname{CSI}(t) \\
& \operatorname{CSR}_{(a c)}=\sum_{1}^{t} \operatorname{CSR}(t)
\end{aligned}
$$


Para el caso propuesto, la $\mathrm{CSI}_{(a c)}$ se reduce un $34 \%$, y la $\operatorname{CSR}_{(a c)}$ se incrementa un $22 \%$, con respecto a la no aplicación del PAPYCS.

TABLA IX

EJ. 1. CS $(j, t, C(t))$ EN (kW), POR PARTICIPANTE $j$ EN EL PERÍODO $t$

\begin{tabular}{cccccc}
\hline \hline$t$ & $C(t)$ & $\mathrm{CS}(1, t, C(t))$ & $\mathrm{CS}(2, t, C(t))$ & $\mathrm{CS}(3, t, C(t))$ & $\mathrm{CS}(4, t, C(t))$ \\
\hline 1 & 5 & 0.75 & 7.75 & 9.50 & -18.00 \\
2 & 3 & 0.75 & 5.75 & 6.50 & -13.00 \\
3 & 3 & 0.75 & 5.75 & 8.50 & -15.00 \\
4 & 1 & 0.75 & 5.75 & 8.50 & -15.00 \\
5 & 1 & 0.75 & 5.75 & 8.50 & -15.00 \\
6 & 3 & 0.75 & 7.25 & 6.50 & -14.50 \\
7 & 3 & 0.75 & 7.25 & 10.00 & -18.00 \\
8 & 1 & 0.75 & 7.25 & 10.00 & -18.00 \\
9 & 1 & 0.75 & 7.25 & 10.00 & -18.00 \\
10 & 3 & 2.00 & 9.25 & 14.00 & -25.25 \\
11 & 1 & 2.00 & 9.25 & 14.00 & -25.25 \\
12 & 2 & 2.00 & 9.25 & 7.00 & -18.25 \\
13 & 2 & 2.00 & 9.25 & 10.00 & -21.25 \\
14 & 1 & 2.00 & 9.25 & 10.00 & -21.25 \\
15 & 1 & 2.00 & 9.25 & 10.00 & -21.25 \\
16 & 2 & 0.25 & 9.25 & 10.00 & -19.50 \\
\hline \hline
\end{tabular}

TABLA X

EJ. 1. $\operatorname{Pr}(j, t, C(t))$ EN (\$A), POR PARTICIPANTE $j$ EN EL PERÍODO $t$

\begin{tabular}{ccccccc}
\hline \hline$t$ & $C(t)$ & $(1, t, C(t))$ & $(2, t, C(t))$ & $(3, t, C(t))$ & $(4, t, C(t))$ & $(s b, t, C(t))$ \\
\hline 1 & 5 & 3.00 & -20.90 & -11.00 & 28.90 & 0.00 \\
2 & 3 & 3.00 & -17.15 & -6.75 & 20.90 & 0.00 \\
3 & 3 & 3.00 & -17.15 & -13.10 & 26.65 & 0.60 \\
4 & 1 & 3.00 & -17.15 & -13.10 & 26.65 & 0.60 \\
5 & 1 & 3.00 & -17.15 & -13.10 & 26.65 & 0.60 \\
6 & 3 & 3.00 & -21.05 & -7.10 & 24.55 & 0.60 \\
7 & 3 & 3.00 & -21.05 & -13.45 & 30.30 & 1.20 \\
8 & 1 & 3.00 & -21.05 & -13.45 & 30.30 & 1.20 \\
9 & 1 & 3.00 & -21.05 & -13.45 & 30.30 & 1.20 \\
10 & 3 & -0.80 & -28.05 & -20.45 & 48.10 & 1.20 \\
11 & 1 & -0.80 & -28.05 & -20.45 & 48.10 & 1.20 \\
12 & 2 & -0.80 & -28.05 & -17.25 & 44.90 & 1.20 \\
13 & 2 & -0.80 & -28.05 & -21.90 & 49.55 & 1.20 \\
14 & 1 & -0.80 & -28.05 & -21.90 & 49.55 & 1.20 \\
15 & 1 & -0.80 & -28.05 & -21.90 & 49.55 & 1.20 \\
16 & 2 & 2.60 & -28.05 & -21.90 & 46.05 & 1.30 \\
\hline \hline
\end{tabular}

En la Fig. 3 se observa que la curva $\operatorname{CST}(t)$ es insensible a las variaciones de $\mathrm{PT}(t)$. En cambio, en la Fig. 4, la curva $\operatorname{CST}(t)$ acompaña a las variaciones de $\operatorname{PT}(t)$. Esto último se debe a que el procedimiento propuesto, apoyado en los datos de entrada, dinamiza el comportamiento de CST $(t)$. Además, el procedimiento permite lograr que $\operatorname{CST}(t)$ se aproxime y siga a $\mathrm{PT}(t)$, en mayor o menor medida, con el solo hecho de alterar los valores asignados en las tablas que forman parte de los datos de entrada. Un comportamiento de la curva $\operatorname{CST}(t)$, que no acompañe y no aproxime a las variaciones de $\operatorname{PT}(t)$ desaprovecha la CS del AP.

En la Tabla $X$ se puede observar el comportamiento del $\operatorname{Pr}(j, t, C(t))$ de cada participante $j$, en el período $t$. Los valores
$\operatorname{Pr}(j, t, C(t))$ se integran con los valores de $\operatorname{CS}(j, t, C(t))$, ponen en conocimiento el pago o cobro que realiza cada participante $j$ en el período $t$, y al mismo tiempo, manifiestan un comportamiento dinámico y dan una idea sobre la importancia que tiene cada participante $j$.

A los efectos de presentar una evaluación económica sencilla, se definen los siguientes indicadores, representativos de los precios totales abonados a lo largo de $\mathrm{T}=16$ períodos, por cada participante $j$ :

$$
\begin{aligned}
& \operatorname{Pr}^{\mathrm{D}}(j)=\sum_{t=1}^{\mathrm{T}} \operatorname{Pr}(j, t, C(t)) \\
& \operatorname{Pr}^{\mathrm{E}}(j)=\mathrm{CS}^{\mathrm{E}}(j) \times \sum_{t=1}^{\mathrm{T}}\left|\frac{\operatorname{Pr}(j, t, C(t))}{\operatorname{CS}(j, t, C(t))}\right|
\end{aligned}
$$

donde $\operatorname{Pr}^{\mathrm{D}}(j)$ y $\operatorname{Pr}^{\mathrm{E}}(j)$ corresponden a las estrategias dinámica (contrato de CS variable) y estática (contrato de CS constante), respectivamente. El indicador $\operatorname{Pr}^{\mathrm{D}}(j)$ considera la suma de todos los precios variables del participante $j$ en los T períodos de análisis. En cambio, $\operatorname{Pr}^{\mathrm{E}}(j)$ asume una $\mathrm{CS}$ constante $\operatorname{CS}^{\mathrm{E}}(j)$ para cada $j$, y un precio promedio por unidad de CS, calculado en base a la evolución observada en el caso dinámico. El índice $\operatorname{Pr}^{\mathrm{E}}(j)$ no es aplicable a la EDE.

En el presente ejemplo, los valores de $\operatorname{CS}^{\mathrm{E}}(j)$ se adoptan iguales a los considerados en la Tabla VI, es decir: $\mathrm{CS}^{\mathrm{E}}(1)=$ $1.0[\mathrm{~kW}] ; \mathrm{CS}^{\mathrm{E}}(2)=7.80[\mathrm{~kW}]$; y CS${ }^{\mathrm{E}}(3)=11.80[\mathrm{~kW}]$. Las valoraciones de los índices se muestran en la Tabla XI. En el caso dinámico, se observa que cada jugador puede recibir o pagar un valor económico, y además esos valores son inferiores a los correspondientes a la estrategia estática.

TABLA XI

EJ. 1. ÍNDICES DE EVALUACIÓN ECONÓMICA (PARA T = 16)

\begin{tabular}{ccc}
\hline \hline$j$ & $\operatorname{Pr}^{\mathrm{D}}(j)$ & $\operatorname{Pr}^{\mathrm{E}}(j)$ \\
\hline$j=1$ & 24.80 & -48.80 \\
$j=2$ & -370.05 & -370.25 \\
$j=3$ & -250.25 & -307.90 \\
$j=4$ (EDE) & 581.00 & $726.95^{(*)}$ \\
$j=s b$ & 14.50 & - \\
\hline \hline (*) Calculado como: $-\left\{\mathrm{CS}^{\mathrm{E}}(1)+\mathrm{CS}^{\mathrm{E}}(2)+\mathrm{CS}^{\mathrm{E}}(3)\right\}$.
\end{tabular}

TABLA XII

EJ. 2. P $(j, t)$ Y CS $(j, t, C(t))$ EN [kW], POR PARTICIPANTE $j$ EN EL PERÍODO $t$

\begin{tabular}{cccccccccc}
\hline \hline$t$ & $\mathrm{P}(4, t)$ & $\mathrm{CS}(4, t, 1)$ & $\mathrm{P}(5, t)$ & $\mathrm{CS}(5, t, 1)$ & $\mathrm{P}(6, t)$ & $\mathrm{CS}(6, t, 1)$ & $\mathrm{PT}(t)$ & $\mathrm{CST}(t)$ \\
\hline 1 & 68.60 & 152.70 & 172.65 & 194.30 & 131.25 & 176.90 & 388.58 & 544.50 \\
2 & 73.76 & 152.70 & 139.90 & 194.30 & 87.70 & 176.90 & 313.27 & 544.50 \\
3 & 48.82 & 152.70 & 128.18 & 194.30 & 108.27 & 176.90 & 297.91 & 544.50 \\
4 & 63.23 & 152.70 & 131.82 & 194.30 & 108.87 & 176.90 & 316.84 & 544.50 \\
5 & 84.19 & 152.70 & 103.78 & 194.30 & 113.10 & 176.90 & 313.30 & 544.50 \\
6 & 63.12 & 152.70 & 154.25 & 194.30 & 90.12 & 176.90 & 320.06 & 544.50 \\
7 & 83.33 & 152.70 & 172.53 & 194.30 & 136.09 & 176.90 & 408.45 & 544.50 \\
8 & 92.66 & 152.70 & 152.54 & 194.30 & 97.25 & 176.90 & 355.61 & 544.50 \\
9 & 112.15 & 152.70 & 139.28 & 194.30 & 107.66 & 176.90 & 372.54 & 544.50 \\
10 & 144.22 & 152.70 & 191.37 & 194.30 & 176.88 & 176.90 & 532.82 & 544.50 \\
11 & 152.67 & 152.70 & 134.83 & 194.30 & 128.13 & 176.90 & 430.51 & 544.50 \\
12 & 102.56 & 152.70 & 133.70 & 194.30 & 96.88 & 176.90 & 345.58 & 544.50 \\
13 & 71.11 & 152.70 & 154.07 & 194.30 & 115.48 & 176.90 & 354.96 & 544.50 \\
14 & 115.37 & 152.70 & 194.30 & 194.30 & 123.31 & 176.90 & 449.68 & 544.50 \\
15 & 117.22 & 152.70 & 159.43 & 194.30 & 125.00 & 176.90 & 417.09 & 544.50 \\
16 & 23.81 & 152.70 & 173.61 & 194.30 & 143.45 & 176.90 & 357.53 & 544.50 \\
\hline \hline
\end{tabular}




\section{B. Ejemplo 2}

Este ejemplo ampliado considera $N=6$ participantes, y una $\operatorname{EDE}(j=7)$ con una CS de $555[\mathrm{~kW}]$. Se mantienen los mismos valores de potencias, CS, TD y TO declarados por los participantes $j=1,2,3$ del Ejemplo 1. La Tabla XII provee la información complementaria necesaria para $j=4,5,6$, y para el caso de la estrategia estática con CS constante.

A continuación se analiza la estrategia dinámica de asignación de CS y precios. La Tabla XIII informa las $\operatorname{TD}\left(j^{c}, t\right)$ y $\mathrm{TO}\left(j^{v}, t\right)$. La Fig. 5 muestra el comportamiento de potencias y CS de todo el sistema. Las Tablas XIV y XV exponen las CS y precios obtenidos con la estrategia dinámica.

TABLA XIII

EJ. 2. $\operatorname{TD}\left(j^{c}, t\right)$ Y TO $\left(j^{v}, t\right)$, POR PARTICIPANTE $j$ EN EL PERÍODO $t$ $X b(\mathrm{~kW})-X s(\mathrm{~kW})-b(\$ a)-s(\$ a)$

\begin{tabular}{cccccccccccc}
\hline \hline \multicolumn{1}{c}{ TD } & \multicolumn{2}{c}{ TO } & \multicolumn{2}{c}{ TD } & \multicolumn{2}{c}{ TO } & \multicolumn{2}{c}{ TD } & \multicolumn{2}{c}{ TO } \\
\multicolumn{2}{c}{$\left(4^{c}, t\right)$} & \multicolumn{2}{c}{$\left(4^{v}, t\right)$} & \multicolumn{2}{c}{$\left(5^{c}, t\right)$} & \multicolumn{2}{c}{$\left(5^{v}, t\right)$} & \multicolumn{2}{c}{$\left(6^{c}, t\right)$} & \multicolumn{2}{c}{$\left(6^{v}, t\right)$} \\
$b$ & $X b$ & $X s$ & $s$ & $B$ & $X b$ & $X s$ & $s$ & $b$ & $X b$ & $X s$ & $s$ \\
\hline 5.70 & 0.3 & 1.0 & 3.75 & 5.65 & 0.3 & 1.0 & 3.65 & 5.80 & 0.3 & 1.0 & 3.80 \\
4.70 & 4.0 & 2.0 & 7.20 & 4.65 & 4.0 & 2.0 & 7.10 & 4.80 & 4.0 & 2.0 & 7.30 \\
3.70 & 7.0 & 3.0 & 8.65 & 3.60 & 7.0 & 3.0 & 8.55 & 3.00 & 7.0 & 3.0 & 8.75 \\
2.20 & 10.0 & 4.0 & 9.70 & 2.10 & 10.0 & 4.0 & 9.60 & 2.30 & 10.0 & 4.0 & 9.80 \\
\hline \hline
\end{tabular}

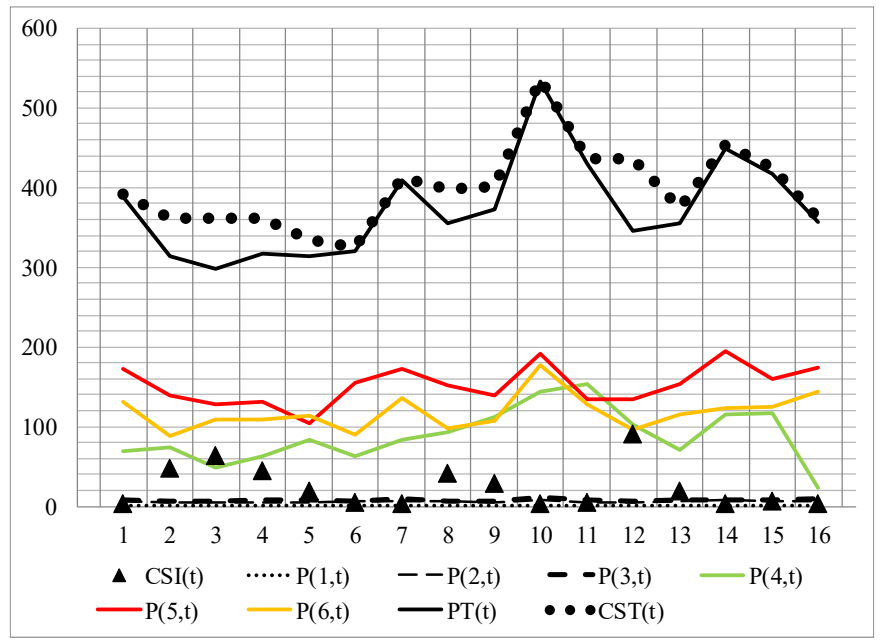

Fig. 5. Ej. 2. Evoluciones de CSs y potencias a lo largo de 16 períodos. Caso de fracciones de CS contratadas variables entre períodos $t$.

Por último, la Tabla XVI presenta los índices de evaluación económica. Para el cálculo de $\operatorname{Pr}^{\mathrm{E}}(j)$ se utilizaron los siguientes valores: $\mathrm{CS}^{\mathrm{E}}(1)=1.0[\mathrm{~kW}] ; \mathrm{CS}^{\mathrm{E}}(2)=7.80$ $[\mathrm{kW}] ; \quad \operatorname{CS}^{\mathrm{E}}(3)=11.80 \quad[\mathrm{~kW}] ; \quad \operatorname{CS}^{\mathrm{E}}(4)=152.70 \quad[\mathrm{~kW}] ;$ $\operatorname{CS}^{\mathrm{E}}(5)=194.30[\mathrm{~kW}] ;$ y CS$^{\mathrm{E}}(6)=176.90[\mathrm{~kW}]$. Al igual que en el Ejemplo 1, todos los participantes logran un beneficio con la estrategia dinámica. Particularmente, los participantes de menor potencia $(\mathrm{j}=1,2,3)$ perciben un ingreso monetario; mientras que la EDE recibe un ingreso inferior al que obtendría con la estrategia estática clásica.
TABLA XIV

EJ. 2. CS $(j, t, C(t))$ EN (kW), POR PARTICIPANTE $j$ EN EL PERÍODO $t$

\begin{tabular}{cclllllll}
\hline \hline$t$ & $C(t)$ & $(1, t, C(t))$ & $(2, t, C(t))$ & $(3, t, C(t))$ & $(4, t, C(t))$ & $(5, t, C(t))$ & $(6, t, C(t))$ & $(7, t, C(t))$ \\
\hline 1 & 87 & 0.75 & 7.10 & 9.25 & 69.40 & 173.20 & 132.45 & -392.15 \\
2 & 19 & 0.75 & 6,45 & 6.75 & 74.70 & 141.20 & 132.45 & -362.30 \\
3 & 2 & 0.75 & 6,45 & 10.25 & 73.70 & 141.20 & 129.95 & -362.30 \\
4 & 1 & 0.75 & 6,45 & 10.25 & 73.70 & 141.20 & 129.95 & -362.30 \\
5 & 22 & 0.75 & 4,50 & 8.25 & 86.15 & 104.20 & 129.95 & -333.80 \\
6 & 24 & 0.75 & 6,70 & 7.10 & 63.65 & 154.65 & 92.45 & -325.30 \\
7 & 30 & 0.75 & 7,10 & 9.45 & 84.55 & 173.70 & 136.65 & -412.20 \\
8 & 13 & 0.75 & 6,45 & 6.95 & 92.85 & 153.70 & 136.65 & -397.35 \\
9 & 13 & 0.75 & 6,45 & 7.45 & 113.25 & 141.05 & 132.65 & -401.60 \\
10 & 34 & 1.35 & 8,45 & 12.75 & 144.85 & 191.75 & 177.20 & -536.35 \\
11 & 15 & 1.35 & 7,15 & 8.75 & 153.15 & 136.75 & 129.20 & -436.35 \\
12 & 1 & 1.35 & 7,15 & 8.75 & 153.15 & 136.75 & 129.20 & -436.35 \\
13 & 30 & 0.55 & 7,15 & 8.75 & 73.15 & 155.70 & 129.20 & -374.50 \\
14 & 18 & 0.95 & 8,50 & 8.75 & 115.40 & 195.00 & 124.70 & -453.30 \\
15 & 9 & 0.95 & 7,85 & 8.75 & 121.55 & 160.00 & 125.35 & -424.45 \\
16 & 54 & 0.20 & 7,85 & 9.70 & 24.40 & 174.25 & 144.45 & -360.85 \\
\hline \hline
\end{tabular}

TABLA XV

EJ. 2. PR $(j, t, C(t))$ EN (\$A), POR PARTICIPANTE $j$ EN EL PERÍODO $t$

\begin{tabular}{lcccccccccc}
\hline \hline \multicolumn{7}{c}{$t C(t)(1, t, C(t))(2, t, C(t))(3, t, C(t))(4, t, C(t))(5, t, C(t))(6, t, C(t))(7, t, C(t))(s b, t, C(t))$} \\
\hline 1 & 87 & 3.00 & -21.90 & -2.15 & -147.80 & -295.85 & -388.45 & 816.55 & 36.60 \\
2 & 19 & 3.00 & -18.90 & 1.35 & -158.80 & -235.05 & -388.45 & 730.55 & 66.30 \\
3 & 2 & 3.00 & -18.90 & -7.95 & -154.15 & -235.05 & -383.80 & 730.55 & 66.30 \\
4 & 1 & 3.00 & -18.90 & -7.95 & -154.15 & -235.05 & -383.80 & 730.55 & 66.30 \\
5 & 22 & 3.00 & -9.90 & -4.15 & -176.35 & -166.65 & -383.80 & 646.55 & 91.30 \\
6 & 24 & 3.00 & 18.35 & -0.35 & -156.75 & -256.85 & -346.75 & 632.90 & 106.45 \\
7 & 30 & 3.00 & 23.55 & -2.50 & -193.15 & -291.80 & -481.35 & 799.65 & 142.60 \\
8 & 13 & 3.00 & 26.55 & 1.00 & -207.95 & -253.80 & -481.35 & 751.15 & 161.40 \\
9 & 13 & 3.00 & 26.55 & 0.45 & -246.00 & -226.15 & -476.50 & 747.15 & 171.50 \\
10 & 34 & 5.80 & 19.55 & 1.65 & -319.65 & -309.70 & -614.25 & 999.80 & 216.80 \\
11 & 15 & 5.80 & 25.55 & 5.30 & -334.45 & -267.90 & -583.85 & 929.60 & 219.95 \\
12 & 1 & 5.80 & 25.55 & 5.30 & -334.45 & -267.90 & -583.85 & 929.60 & 219.95 \\
13 & 30 & 11.00 & 25.55 & 5.30 & -273.65 & -314.70 & -583.85 & 904.10 & 226.25 \\
14 & 18 & 13.00 & 19.25 & 5.30 & -356.55 & -378.80 & -570.95 & 1021.50 & 247.25 \\
15 & 9 & 13.00 & 22.85 & 5.30 & -363.95 & -352.20 & -574.65 & 1002.10 & 247.55 \\
16 & 54 & 17.65 & 22.85 & 3.75 & --179.50 & -380.30 & -608.20 & 788.55 & 335.20 \\
\hline \hline
\end{tabular}

TABLA XVI

EJ. 2. ÍNDICES DE EVALUACIÓN ECONÓMICA (PARA T $=16$ )

\begin{tabular}{ccc}
\hline \hline$j$ & $\operatorname{Pr}^{\mathrm{D}}(j)$ & $\operatorname{Pr}^{\mathrm{E}}(j)$ \\
\hline$j=1$ & 99.05 & -184.51 \\
$j=2$ & 167.65 & -385.97 \\
$j=3$ & 9.65 & -77.28 \\
$j=4$ & -3757.30 & -6627.25 \\
$j=5$ & -4467.75 & -5591.47 \\
$j=6$ & -7833.85 & -10527.41 \\
$j=7$ (EDE) & 13160.85 & $23393.90^{(*)}$ \\
$j=s b$ & 2621.70 & - \\
\hline \hline$\left.{ }^{*}\right)$ Calculado como: $-\left\{\mathrm{CS}^{\mathrm{E}}(1)+\mathrm{CS}^{\mathrm{E}}(2)+\cdots+\mathrm{CS}^{\mathrm{E}}(6)\right\}$
\end{tabular}

\section{Datos Fuente y Plataforma de Experimentación}

Los datos de potencias y CS utilizados en el Ejemplo 1 corresponden a tres unidades de consumo ubicadas en la ciudad de Rafaela (Argentina). Los datos del Ejemplo 2 
correspondientes a los participantes $j=4,5,6$ se obtuvieron mediante un escalado de la información del Ejemplo 1.

Todos los algoritmos de este trabajo se implementaron en el ambiente Matlab ${ }^{\mathrm{TM}}$, utilizándose una computadora portátil (procesador AMD E-350, 1.6 GHz, 3 GB RAM). Los tiempos simulación, por período fueron del orden de 0.1 segundos para el Ejemplo 1 (4 jugadores), y de 0.2 segundos para el Ejemplo 2 (7 jugadores).

\section{CONCLUSIONES}

Se estudiaron las complejas interacciones que pueden existir cuando se procura comercializar CS entre un conjunto de empresas conectadas a un mismo sistema de distribución eléctrica, con energía provista por una única distribuidora. En esta propuesta, mediante un juego No Cooperativo, cada participante define estratégicamente la fracción de CS que está dispuesto a vender o comprar, para: 1) minimizar la $\operatorname{CSI}(t), 2)$ maximizar la $\operatorname{CSR}(t)$, y 3$)$ no ser penalizado. Los precios de negociación en el mercado de CS se determinan mediante un enfoque basado en SD.

Los ejemplos de simulación permitieron comparar estrategias estáticas y dinámicas de asignación de CS y precios. En el primer caso, las CS contratadas constantes originaron precios más elevados que los correspondientes a la estrategia dinámica. En esta última, la reducción de precios se logró gracias a la posibilidad de admitir variabilidad en la CS contratada en cada período. Además, la seguridad de los datos de la transacción queda determinada por la relación biunívoca existente entre precio-participante, dado que no se originan dos valores monetarios iguales en ventas o compras.

La estrategia propuesta puede ser útil como base para el posterior desarrollo de un modelo de juego dinámico más elaborado, en el cual los jugadores puedan tomar decisiones a largo plazo con respecto a sus procesos de comercialización de fracciones de CS.

\section{APÉNDICE: NOMENCLATURA}

Siglas Principales

AlgSD Algoritmo de SD.

AlgACS Algoritmo para asignar las fracciones de CS.

AlgMET Algoritmo para mejorar la eficiencia de las transacciones.

AP Alimentador principal.

CS Capacidad de suministro.

PAPYCS Procedimiento de asignación de precios y de fracción de CS.

SD Subasta doble.

TD, TO Tablas de demanda y oferta.

Variables

$b_{(q)}\left(j^{c}, t\right) \quad$ Precio ofrecido para la compra de CS por el participante $j^{c}$ en su $q$-ésima oferta durante el período $t$.

$\operatorname{CS}(j, t) \quad$ CS del participante $j$ en el período $t$.

$\operatorname{CS}(j, t, c) \quad$ CS del participante $j$ en el ciclo $c$ del período $t$.

$\operatorname{CS}(t) \quad \mathrm{CS}$ de la EDE a través del AP, en el período $t$.

$\operatorname{CSI}(t) \quad$ CS inactiva, en el período $t$ (Ec. (15)).

$\operatorname{CST}(t) \quad$ CS total contratada a la EDE, en el período $t$.

$\operatorname{CSR}(t) \quad$ CS remanente, en el período $t$.

$c \quad$ Ciclo de ejecución del PAPYCS, $\operatorname{con} c=1, \ldots, C(t)$.

$i \quad$ Posición de la pareja vendedor-comprador (en la Tabla III).

$j \quad$ Agente participante en la SD, con $j=1, \ldots, J \leq N+1$.

$j^{c}, j^{v} \quad$ Agente participante como comprador o vendedor.

$\mathrm{k} \quad$ Número de transacciones posibles de compra-venta. $n \quad$ Industria $n$-ésima, con $n=1, \ldots, N(n \equiv j)$.

$\mathrm{P}(j, t) \quad$ Potencia demandada por el participante $j$, en el período $t$.

$\operatorname{Pr}_{0}(t, c) \quad$ Precio de Negociación, en el ciclo $c$ del período $t$.

$\operatorname{Pr}^{\mathrm{D}}(j) \quad$ Índice económico para contratos dinámicos (Ec. (19))

$\operatorname{Pr}^{\mathrm{E}}(j) \quad$ Índice económico para contratos estáticos (Ec. (20))

$\operatorname{Pr}(j, t) \quad$ Precio asignado al participante $j$ en el período $t$.

$\operatorname{Pr}(j, t, c) \quad$ Precio asignado al participante $j$ en el ciclo $c$ del período $t$.

$\operatorname{Pr}\left(s b, t_{0}\right) \quad$ Precio asignado al subastador correspondiente al período $t_{0}$.

$\operatorname{Pr}(s b, t, c)$ Precio asignado al subastador en el ciclo $c$ del período $t$.

$\operatorname{PT}(t) \quad$ Potencia total demandada a la EDE, en el período $t$.

$s_{(w)}\left(j^{v}, t\right) \quad$ Precio ofrecido para la venta de CS por el participante $j^{v}$ en su $w$-ésima oferta durante el período $t$.

$\operatorname{TD}(j, t) \quad$ Tabla de Demanda, del participante $j$ en el período $t$.

TO $(j, t) \quad$ Tabla de Oferta, del participante $j$ en el período $t$.

$t, t_{0} \quad$ Período de tiempo de análisis $(t)$ siguiente al período actual $\left(t_{0}\right)$.

$u^{c}(j, t, c)$ Número de veces que interviene el participante $j$ como comprador (Tabla V), en el ciclo $c$ del período $t$.

$u^{v}(j, t, c) \quad$ Número de veces que interviene el participante $j$ como vendedor (Tabla V), en el ciclo $c$ del período $t$.

$v(j) \quad$ Número total de participaciones de $j^{v}$ (en la Tabla III).

$X b_{(q)}\left(j^{c}, t\right)$ Fracción de CS demandada por el participante $j^{c}$ en su $q$-ésima oferta durante el período $t$.

$X s_{(w)}\left(j^{v}, t\right)$ Fracción de CS ofrecida por el participante $j^{v}$ en su $w$-ésima oferta durante el período $t$.

$X P_{(i)}(t, c) \quad$ Fracción de CS promedio (posición $i$ ), en el ciclo $c$ del período $t$.

Vectores y Matrices

$\mathbf{M}_{j} \quad$ Matriz de las $X P_{(i)}(t, c)$ correspondientes al vendedor $j$.

$\mathbf{r}_{j} \quad$ Vector diferencia calculado como: $\operatorname{vec}\{\Delta \operatorname{CS}(j, t, c)\}-\mathbf{s}_{j}$.

$\mathbf{s}_{j} \quad$ Vector que contiene las sumas de las filas de $\mathbf{M}_{j}$.

Variables Griegas

$\Delta \mathrm{CS}(j, t, c)$ Variación de CS, del participante $j$ en el ciclo $c$ del período $t$. $\Delta \mathrm{CS}^{c}\left(j^{c}, t, c\right)$ Variación promedio de CS, de $j^{c}$ en el ciclo $c$ del período $t$. $\Delta \operatorname{CS}^{v}\left(j^{v}, t, c\right)$ Variación promedio de CS, de $j^{v}$ en el ciclo $c$ del período $t$.

Supra-índices y Sub-índices

$c, v \quad$ Comprador y vendedor, respectivamente.

(ac) Acumulado en todos los períodos.

\section{AGRADECIMIENTOS}

Los autores agradecen a la Universidad Tecnológica Nacional (UTN), Facultades Regionales Rafaela y Santa Fe, por el apoyo recibido mediante proyectos de investigación. A. Rocchi agradece a la UTN por el financiamiento recibido a través de su beca de Doctorado. J. Vega agradece el apoyo brindado por la red temática CYTED 717RT0533 MEIHAPER (Microrredes Eléctricas Inteligentes Híbridas con Alta Penetración de Energías Renovables).

\section{REFERENCIAS}

[1] J. D. Molina, H. Rudnick, "Transmission of electric energy: A bibliographic review", IEEE Latin America Transactions, vol. 8, no. 3, pp. 245-258, 2010.

[2] W. W. Weaver and P. T. Krein, "Game-theoretic control of small scale power systems," IEEE Trans. Power Del., vol. 24, no. 3, pp. 1560-1567, 2009.

[3] E. Baeyens, E. Y. Bitar, P. P. Khargonekar, and K. Poolla, "Wind energy aggregation: A coalitional game approach," in Proc. 50th IEEE Conf. Decision Control (CDC), Orlando, FL, USA, Dec. 2011.

[4] X. Z. Xu and Q. Chen, "Research on reactive power compensation algorithm based on game theory in wind farm," Key Eng. Mater., vol. 439, pp. 989-993, 2010. 
[5] M. S. Chamba, O. Añó, "Economic dispatch of energy and reserve in competitive markets using meta-heuristic algorithms," IEEE Latin America Transactions, vol. 11, no. 1, pp. 473-478, 2013.

[6] J. C. Silva Chavez, A. Zamora-Mendez, M. R. Arrieta Paternina, J. F. Yrena Heredia, R. Cardenas-Javier, "A hybrid optimization framework for the non-convex economic dispatch problem via meta-heuristic algorithm", Electr. Power Syst. Res., vol. 177, 105999, 2019.

[7] B. F. Hobbs, C. B. Metzler, and J.-S. Pang, "Strategic gaming analysis for electric power systems: An MPEC approach," IEEE Trans. Power Syst., vol. 15 , no. 2 , pp. 638-645, 2000.

[8] S. K. Ng, C. Lee, and J. Zhong, "A game-theoretic approach to study strategic interaction between transmission and generation expansion planning," in Proc. 38th North Amer. Power Symp., 2006, pp. 115-120.

[9] E. Bompard, W. Lu, and R. Napoli, "Network constraint impacts on the competitive electricity markets under supply-side strategic bidding," IEEE Trans. Power Syst., vol. 21, no. 1, pp. 160-170, 2006.

[10] W. El-Baz, P. Tzscheutschler, and U. Wagner, "Integration of energy markets in microgrids: A double-sided auction with device-oriented bidding strategies". Applied Energy 241, pp. 625-639, 2019.

[11] W. Saad, Z. Han, H.V. Poor, and T. Başar, "A noncooperative game for double auction-based energy trading between PHEVs and distribution grids," in Proc. IEEE Int. Conf. Smart Grid Commun. (Smart-GridComm.), Brussels, Belgium, Oct. 2011.

[12] P. R. Wurman, W. E. Walsh, and M. P. Wellman, "Flexible double auctions for electronic commerce: Theory and implementation," Decision Support Systems, vol. 24, pp. 17-27, 1998.

[13] B. H. Zaidi and S. H. Hong, "Combinatorial double auctions for multiple microgrid trading", Electrical Engineering, pp. 1-15, 2017.

[14] J. Wang, Q. Wang, N. Zhou, and Y. Chi, "A Novel Electricity Transaction Mode of Microgrids Based on Blockchain and Continuous Double Auction”. Energies, 10. 1971, 2017.

[15] S. Thakur, B. P. Hayes, and J. G. Breslin, "Distributed Double Auction for Peer to Peer Energy Trade using Blockchains", 5th Int. Symp. on Environment-Friendly Energies and Applications (EFEA), 2018.

[16] M. Yokoo, Y. Sakurai, and S. Matsubara, "Robust double auction protocol against false-name bids," Proc. 21st Intern. Conf. on Distributed Computing Systems, Publisher: IEEE, pp. 137-145, 2001.

[17] B. P. Majumder, M. N. Faqiry, S. Das, and A. Pahwa, "An efficient iterative double auction for energy trading in microgrids", 2014 IEEE Symp. on Computational Intelligence Applications in Smart Grid (CIASG), 2014.

[18] N. Wang, W. Xu, W. Shao, and Z. Xu, "A Q-Cube Framework of Reinforcement Learning Algorithm for Continuous Double Auction among Microgrids". Energies, 12. 2891, 2019.

[19] R. P. McAfee, "A dominant strategy double auction," Journal of Economic Theory, vol. 56, pp. 434-450, 1992.

[20] N. Rotering and M. Ilic, "Optimal charge control of plug-in hybrid electric vehicles in deregulated electricity markets," IEEE Trans. Power Syst., vol. 26, no. 3, pp. 1021-1029, 2011.

[21] N. Lu, J. H. Chow, and A. A. Desrochers, "Pumped-storage hydroturbine bidding strategies in a competitive electricity market," IEEE Trans. Power Syst., vol. 19, no. 2, pp. 834-841, 2004.

[22] Y. Wang, W. Saad, Z. Han, H. V. Poor, and T. Basar, "A GameTheoretic Approach to Energy Trading in the Smart Grid," IEEE Trans. on Smart Grid, vol. 5, no. 3, pp. 1439-1450, 2014.

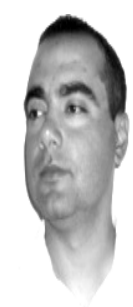

Ariel Mariano Rocchi es Ingeniero Electromecánico (2004) y Magíster en Administración de Negocios (2014) por la Universidad Tecnológica Nacional (UTN) Facultad Regional Rafaela, Argentina. Con experiencia en Evaluación de Proyectos de Inversión, y Soldadura por: Arco Transferido, Plasma, Fricción y Proyección y tiene interés en los temas de Redes Eléctricas Inteligentes y Energías Renovables.

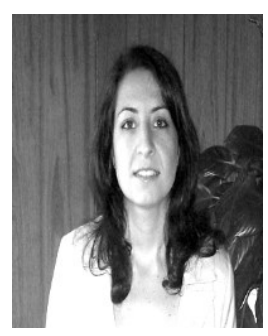

Érica Soledad Fernández es Ingeniera Industrial (UTN, Facultad Regional Santa Fe, FRSF), Doctora en Ingeniería en Sistemas de Información (UTN, FRSF), y Profesora Adjunto de Ingeniería Industrial de la UTN desde 2007. Actualmente, su principal área de investigación es Informática Industrial, específicamente el desarrollo de herramientas soportes de decisión para microrredes eléctricas con incorporación de energías renovables, cadenas de suministros y procesos productivos.

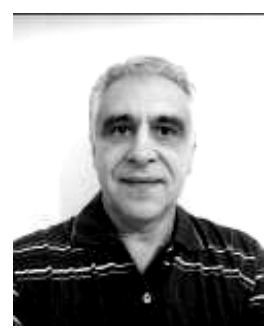

Jorge Ruben Vega egresó en 1985 como Ingeniero Electricista (Univ. Nac. de La Plata, UNLP, Argentina), y en 1993 como Doctor en Tecnología Química (Univ. Nac. del Litoral, UNL, Santa Fe, Argentina). Actualmente, es Profesor Titular en el Departamento de Ingeniería Eléctrica y Director del Centro de Investigación y Desarrollo en Ingeniería Eléctrica y Sistemas Energéticos - CIESE (UTN, FRSF), e Investigador Principal del CONICET en el INTEC (CONICET-UNL). 